\title{
First Direct Mass Measurements of Nuclides around $Z=100$ with a Multireflection Time-of-Flight Mass Spectrograph
}

\author{
Y. Ito, ${ }^{1,}{ }^{*}$ P. Schury, ${ }^{2}$ M. Wada, ${ }^{1,2}$ F. Arai,,${ }^{1,3}$ H. Haba, ${ }^{1}$ Y. Hirayama, ${ }^{2}$ S. Ishizawa, ${ }^{1,4}$ D. Kaji, ${ }^{1}$ S. Kimura, ${ }^{1,2,3}$ H. Koura, ${ }^{5}$ \\ M. MacCormick, ${ }^{6}$ H. Miyatake, ${ }^{2}$ J. Y. Moon, ${ }^{2,7}$ K. Morimoto, ${ }^{1}$ K. Morita, ${ }^{1,8}$ M. Mukai, ${ }^{2,3}$ I. Murray, ${ }^{1}$ T. Niwase, ${ }^{8}$ \\ K. Okada, ${ }^{1,9}$ A. Ozawa, ${ }^{3}$ M. Rosenbusch, ${ }^{1}$ A. Takamine, ${ }^{1}$ T. Tanaka, ${ }^{1,8}$ Y. X. Watanabe, ${ }^{2}$ H. Wollnik, ${ }^{1,10}$ and S. Yamaki ${ }^{1,11}$ \\ ${ }^{1}$ RIKEN Nishina Center for Accelerator-Based Science, Wako 351-0198, Japan \\ ${ }^{2}$ Wako Nuclear Science Center (WNSC), Institute of Particle and Nuclear Studies (IPNS), \\ High Energy Accelerator Research Organization (KEK), Wako 351-0198, Japan \\ ${ }^{3}$ University of Tsukuba, Tsukuba, Ibaraki 305-8577, Japan \\ ${ }^{4}$ Graduate School of Science and Engineering, Yamagata University, Yamagata 990-8560, Japan \\ ${ }^{5}$ Japan Atomic Energy Agency, Tokai, Ibaraki 319-1185, Japan \\ ${ }^{6}$ Institut de Physique Nucléaire, IN2P3-CNRS, Université Paris-Sud, Université Paris-Saclay, 91406 Orsay Cedex, France \\ ${ }^{7}$ Rare Isotope Science Project, Institute for Basic Science (IBS), Daejeon 305-811, Korea \\ ${ }^{8}$ Department of Physics, Kyushu University, Nishi-ku, Fukuoka 819-0395, Japan \\ ${ }^{9}$ Sophia University, Chiyoda-ku, Tokyo 102-8554, Japan \\ ${ }^{10}$ Department of Chemistry and Biochemistry, New Mexico State University, Las Cruces, New Mexico 88003, USA \\ ${ }^{11}$ Department of Physics, Saitama University, Sakura-ku, Saitama 338-8570, Japan
}

(Received 29 September 2017; revised manuscript received 5 February 2018; published 10 April 2018)

The masses of ${ }^{246} \mathrm{Es},{ }^{251} \mathrm{Fm}$, and the transfermium nuclei ${ }^{249-252} \mathrm{Md}$ and ${ }^{254} \mathrm{No}$, produced by hot- and cold-fusion reactions, in the vicinity of the deformed $N=152$ neutron shell closure, have been directly measured using a multireflection time-of-flight mass spectrograph. The masses of ${ }^{246} \mathrm{Es}$ and ${ }^{249,250,252} \mathrm{Md}$ were measured for the first time. Using the masses of ${ }^{249,250} \mathrm{Md}$ as anchor points for $\alpha$ decay chains, the masses of heavier nuclei, up to ${ }^{261} \mathrm{Bh}$ and ${ }^{266} \mathrm{Mt}$, were determined. These new masses were compared with theoretical global mass models and demonstrated to be in good agreement with macroscopic-microscopic models in this region. The empirical shell gap parameter $\delta_{2 n}$ derived from three isotopic masses was updated with the new masses and corroborates the existence of the deformed $N=152$ neutron shell closure for Md and Lr.

DOI: 10.1103/PhysRevLett.120.152501

Precision mass measurements of unstable nuclei, providing a direct measure of the nuclear binding energy, are invaluable for the study of nuclear shell evolution and collective effects, such as deformations, far from stability $[1,2]$. For transfermium nuclei and the yet poorly investigated region towards the superheavy nuclei (SHN), where proton repulsion becomes a generally dominant feature, the description of nuclear lifetimes depends crucially on shell stabilization effects mainly driven by deformed shells [3-5]. Theoretical studies with increasing particle numbers investigate the so-called "island of stability" [6], where features like the continuing decrease of energy gaps [7] and the emergence of shape coexistence [8] have a crucial impact on the predicted position and localization of stability regions and the corresponding lifetimes of the nuclei. Although the

Published by the American Physical Society under the terms of the Creative Commons Attribution 4.0 International license. Further distribution of this work must maintain attribution to the author(s) and the published article's title, journal citation, and DOI. first experimental evidence for SHN has reached the region of the predicted subshell closure at $N=162$ [9-11], the deformed shell closure at $N=152$ for transfermium nuclei (see, e.g., [12]) and, as recently pointed out, weaker shell effects in the vicinity [13], still represents the cutting edge for thorough experimental investigations. The transfermium nuclei, however, can be produced only online, in heavy-ion fusion and nucleon transfer reactions, and consequently only low yields are available for study, necessitating highly efficient techniques. Direct mass measurements of transfermium nuclei have so far been performed for only six nuclei-four isotopes of nobelium and two isotopes of lawrencium-with the Penning trap mass spectrometer SHIPTRAP $[14,15]$.

In this Letter, we report the first implementation of a multireflection time-of-flight mass spectrograph (MRTOF MS) for transfermium nuclei as shown in Fig. 1, including new mass measurements of ${ }^{246} \mathrm{Es},{ }^{251} \mathrm{Fm}$, ${ }^{249-252} \mathrm{Md}$, and ${ }^{254} \mathrm{No}$, performed with sub-parts-per-million precision. This represents the first determination of the masses of ${ }^{249-250,252} \mathrm{Md}$, closing a gap of unmeasured nuclei which could not be linked by corresponding decay chains. 


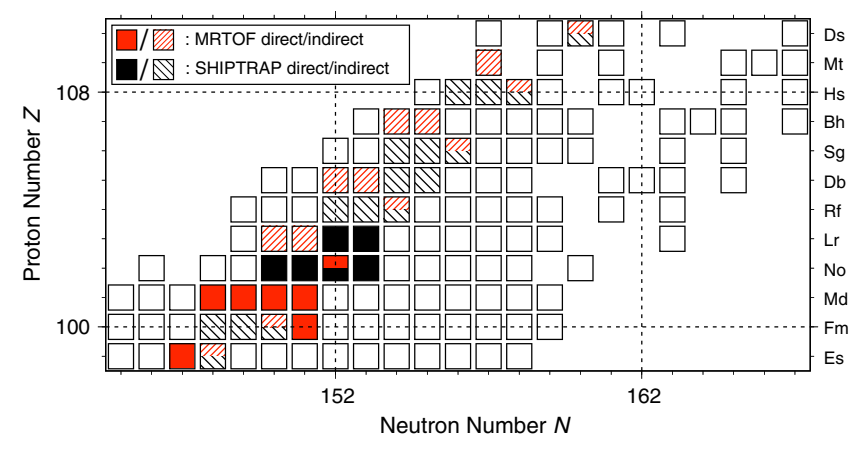

FIG. 1. Nuclear chart above californium $(Z=98)$. The squares indicate nuclei synthesized so far. Nuclei whose masses were determined in this work are indicated by the solid (direct) and the left-hatched (indirect) red squares. Similarly, nuclei whose masses were determined by SHIPTRAP measurements are indicated by the solid (direct) and the right-hatched (indirect) black squares.

Through combining the masses of the dominantly $\beta$-decaying nuclei ${ }^{249-250} \mathrm{Md}$ with previously known $Q_{\alpha}$ values, the masses of nuclei up to ${ }^{261} \mathrm{Bh}$ and ${ }^{266} \mathrm{Mt}$ could be experimentally determined for the first time. These results support the existence of the $N=152$ shell gap in $\mathrm{Md}$ and $\mathrm{Lr}$ while also providing the first experimental data at the shell gap for Db isotopes.

As shown in Fig. 2, the MRTOF MS [16,17] was installed behind a cryogenic helium gas cell and ion trap system coupled with the gas-filled recoil ion separator GARIS-II [18]. Primary beams provided by the RIKEN heavy-ion linear accelerator RILAC impinged upon a rotating target to produce fusion-evaporation residues (ER). The stopping of high-energy ER in the gas cell was optimized by adjusting the thickness of a Mylar degrader while the gas cell was filled with 150 mbar helium at a temperature of $150 \mathrm{~K}$. The ions were transported to a radio frequency carpet (RFC) [19], located on the exit wall, by a static electric field and then extracted by means of a traveling-wave (TW) mode RFC technique [20-22]. The extracted ions were transported through a differential pumping section by a sextupole ion guide (SPIG) and then accumulated in the first ion trap system. After accumulating and cooling in the flat trap, ion bunches were orthogonally ejected, accelerated to a kinetic energy of $\approx 1.7 \mathrm{keV}$ by a pulsed drift tube (Acc-PDT), transported through an electrostatic multiple lens and a BradburyNielsen gate (BN gate) [23], and decelerated to tens of electron volts by a second pulsed drift tube (Dec-PDT) before being retrapped in the second ion trap system, located in the experimental room underneath GARIS-II.

The first and second ion trap systems, each consisting of a pair of linear Paul traps on either side of a "flat" ion trap [24], have the same geometry and were filled with helium buffer gas at $\sim 10^{-2}$ mbar. In the first trap system, the fore and aft linear Paul traps accumulated the continuous ion beams from the gas cell and from a thermal ion source,

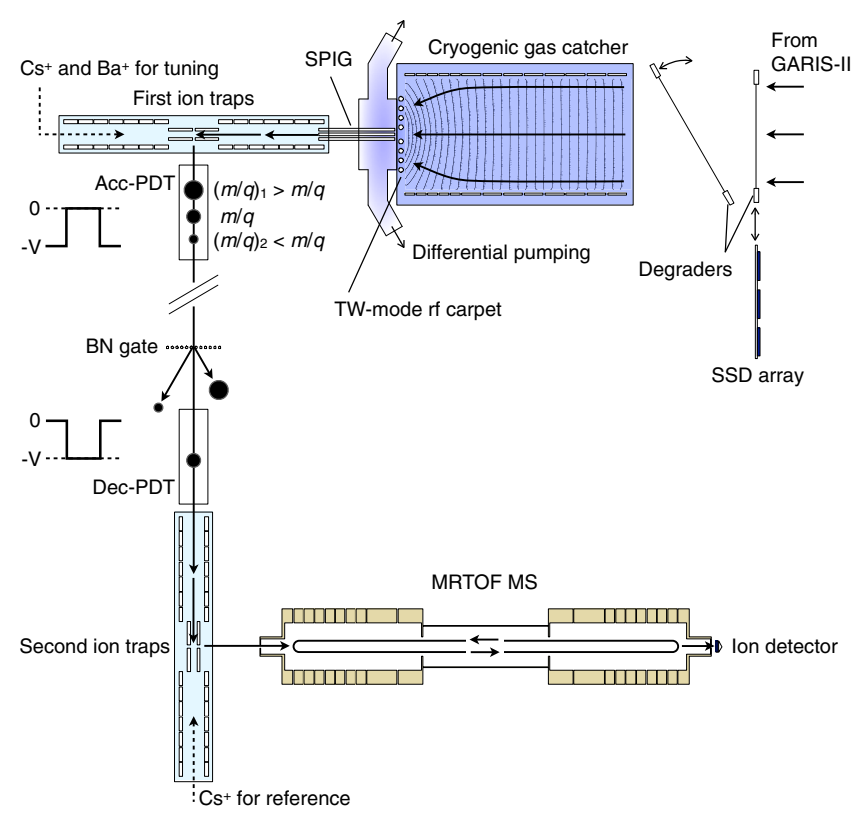

FIG. 2. Schematic view of the experimental setup. Dashed and solid arrows schematically depict the paths of reference and analyte ions, respectively. Curved lines in the cryogenic gas cell represent equipotential lines of the static field that focus ions onto the rf carpet, while arrows depict the nominal trajectories of ions in the static field.

respectively, in order to precool and prebunch the ion beam prior to transfer to the flat trap. The thermal ion source in the first trap system provided both $\mathrm{Cs}^{+}$and $\mathrm{Ba}^{+}$ions for beam-line tuning. In the second trap system, the fore and aft linear Paul traps accumulated and precooled the pulsed beam delivered from the first trap and the continuous beam from a thermal reference ion source, respectively. The second trap system's thermal ion source provided reference $\mathrm{Cs}^{+}$ions for the mass measurements.

The novel flat trap geometry allowed the implementation of a concomitant measurement scheme, shown in Fig. 3(a).

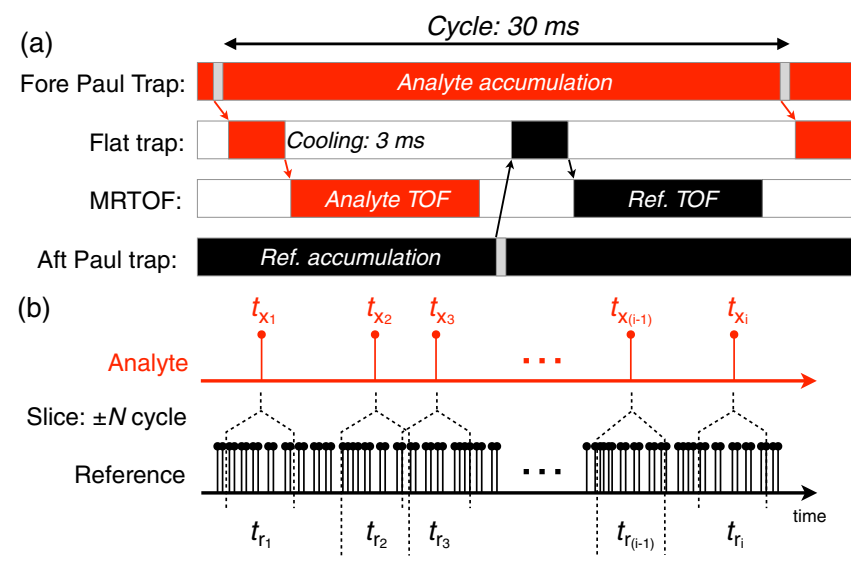

FIG. 3. (a) Pictorial representations of the concomitant referencing scheme in the second ion trap system. (b) Slice-by-event analysis method. 
While ions from the gas cell were being analyzed with the MRTOF MS, reference ions stored in the second trap system's aft linear Paul trap were transferred to the flat ion trap and cooled, while pulses of ions sent from the first trap system continue to accumulate in the lower trap system's fore linear Paul trap. In this way, measurements of analyte ions from the gas cell were interleaved with measurements of reference ions within a $30 \mathrm{~ms}$ cycle (15 ms for each). In addition to providing a nearly $100 \%$ duty cycle, the times of flight (TOF) of the reference ions provide precise corrections of TOF drifts for both reference ions and analyte ions.

The TOF drift correction was performed in a manner we refer to as the "slice-by-event" method [see Fig. 3(b)]. Because analyte detection events were sparse, it was not necessary to consider all reference events. Rather, the reference ions detected 50 cycles $(1.5 \mathrm{~s})$ before and after each analyte detection event were combined to produce a reference spectrum. The centroid of reference events in each slice was used to determine the reference TOF $t_{r_{i}}$ for each analyte TOF $t_{x_{i}}$. Drift-corrected spectra can then be produced for the reference and analyte by multiplying the TOF of each detected ion in subset $i$ by $t_{r_{0}} / t_{r_{i}}$. A detailed review of this analytical method will be provided in a future publication. Spectra were fitted with an unbinned maximum-likelihood estimator using an asymmetric combined Gaussian-Lorentzian function [25].

Because of the multireflection nature of the MRTOF MS, there is not a one-to-one correspondence between the TOF and $A / q$; unambiguous identification cannot be made from a single spectrum. This is a consequence of the possibility that two ion species differing in the mass-to-charge ratio by $\Delta A / q$ will also differ in the number of laps made in the MRTOF MS by $\Delta n$ laps such that they have essentially the same TOF. To avoid misidentifications, therefore, we employed confirmation measurements of each analyte ion at different numbers of laps (generally, \pm 1 laps) and additionally for low count-rate measurements of ${ }^{249,250} \mathrm{Md}$ further confirmations with a dummy target of lower $Z$, which is unable to produce the desired ER but that can be presumed to provide otherwise similar conditions. Figure 4 demonstrates this process in the case of ${ }^{250} \mathrm{Md}$. After $6000 \mathrm{~s}$, within $\pm 50 \mathrm{~ns}$ of the expected TOF of ${ }^{250} \mathrm{Md}^{2+}$ seven and five counts, respectively, were observed at $n=144$ and $n=145$ laps while using nat Tl $(Z=81)$ targets; no counts were observed when using ${ }^{197} \mathrm{Au}$ $(Z=79)$ targets in $4000 \mathrm{~s}$ for both. This provides strong evidence that the observed spectral peak truly belongs to ${ }^{250} \mathrm{Md}^{2+}$ with the probability of no detected events being only $0.03 \%$. The raw and binned spectrum observed for ${ }^{250} \mathrm{Md}$ at $n=145$ laps, along with the resultant fitting curve by an unbinned maximum-likelihood routine [26], is shown in Fig. 5. This process was employed for each isotope measured.

Experimental conditions, reactions, and primary beam energies for each measured isotope are included in Table I. ${ }^{246} \mathrm{Es},{ }^{251} \mathrm{Fm}$, and ${ }^{252} \mathrm{Md}$ were produced with hot-fusion

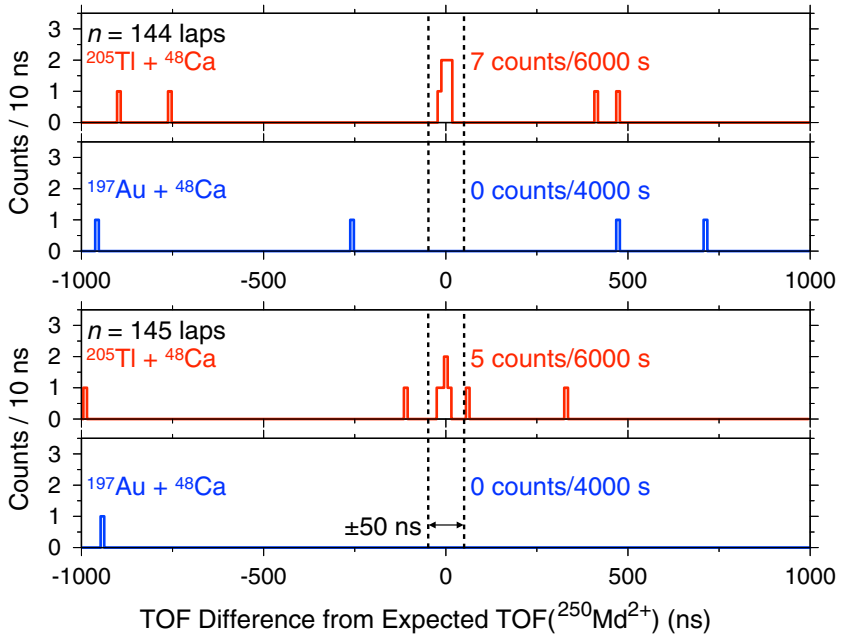

FIG. 4. TOF spectra in the anticipated vicinity of ${ }^{250} \mathrm{Md}^{2+}$ at $n=144$ and 145 laps for ${ }^{\text {nat }} \mathrm{Tl}$ and ${ }^{197} \mathrm{Au}$ targets.

reactions using ${ }^{18} \mathrm{O}$ and ${ }^{19} \mathrm{~F}$ primary beams with intensities of $\sim 3 \mathrm{p} \mu \mathrm{A}$ on ${ }^{232} \mathrm{Th}$ and ${ }^{\text {nat }} \mathrm{U}$ targets. $\mathrm{A}{ }^{48} \mathrm{Ca}$ primary beam of $\sim 3 \mathrm{p} \mu \mathrm{A}$ intensity was used to produce ${ }^{249,250,251} \mathrm{Md}$ and ${ }^{254} \mathrm{No}$ via cold-fusion reactions with ${ }^{\text {nat }} \mathrm{Tl}$ and ${ }^{208} \mathrm{~Pb}$ targets. All targets had a thickness of $\sim 500 \mu \mathrm{g} / \mathrm{cm}^{2}$ with $1.4-\mathrm{mg} / \mathrm{cm}^{2} \mathrm{Ti}$ backings for actinide targets and $60-\mu \mathrm{g} / \mathrm{cm}^{2} \mathrm{C}$ backing for other targets and were mounted on a $300-\mathrm{mm}$ wheel [27] which rotated at $2000 \mathrm{rpm}$ during irradiation.

Results for all isotopes measured are listed in Table I; the mass values are compared with AME16 [33] values in Fig. 6. The masses were derived using the single-reference analysis method described in Ref. [16]. The listed systematic uncertainties derive from ambiguity in the origin of the time of flight. As expected from the short measurement cycle, TOF spectra for ${ }^{254}$ No included a $~ 30 \%$ admixture [34] of the 1.295(2) MeV isomer. While the isomer and ground state could be only partially resolved, the mass of ${ }^{254 \mathrm{~g}} \mathrm{No}$ is consistent with prior direct measurements at the Penning trap mass spectrometer SHIPTRAP [14]. Furthermore, the masses of ${ }^{251} \mathrm{Fm}$ and ${ }^{251} \mathrm{Md}$ are in good agreement with those determined by $Q_{\alpha}$ [28] using

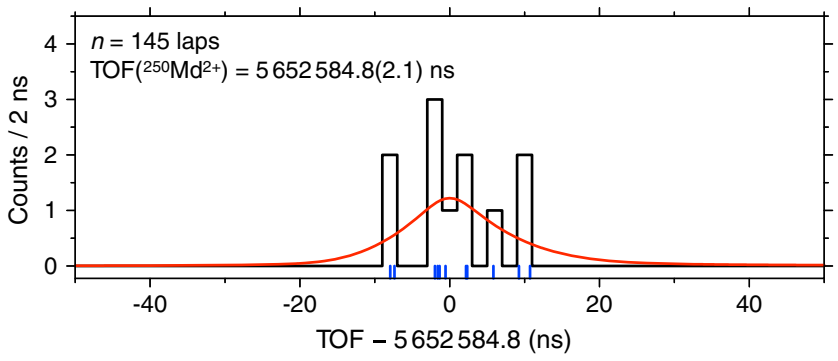

FIG. 5. Fitted TOF spectrum of ${ }^{250} \mathrm{Md}^{2+}$ at $n=145$ laps. The shape parameters of the fitting function were predetermined with a high statistics reference $\left({ }^{133} \mathrm{Cs}^{+}\right)$peak in the same measurement, at the same number of laps. 
TABLE I. Measured isotopes, reactions, reaction energies at the target center in the laboratory frame $\left(E_{\text {lab }}\right)$, recoil energies $\left(E_{\text {recoil }}\right)$, cross sections $\left(\sigma_{\mathrm{ER}}\right)$, squares of the analyte-reference TOF ratio $\left(\rho^{2}\right)$, mass excesses from this work $\left(\mathrm{ME}_{\mathrm{MRTOF}}\right)$ and from the atomic mass evaluation 2016 (AME16) $\left(\mathrm{ME}_{\mathrm{AME16}}\right)$ [28], mass deviations ( $\Delta m=\mathrm{ME}_{\mathrm{MRTOF}}-\mathrm{ME}_{\mathrm{AME16}}$ ), and the total number of detected ions $\left(N_{\text {ion }}\right)$ in this work. Parenthetical values of $\sigma_{\mathrm{ER}}$ denote estimated values from a Monte Carlo code [29]. Extrapolated values of $\mathrm{ME}_{\mathrm{AME} 16}$ are denoted by \#. Experimental statistical and systematic uncertainties are described in the first and second parentheses in $\mathrm{ME}_{\mathrm{MRTOF}}$ and $\Delta m$, respectively.

\begin{tabular}{lccccccccc}
\hline \hline Isotope & Reaction & $\begin{array}{c}E_{\text {lab }} \\
(\mathrm{MeV})\end{array}$ & $\begin{array}{c}E_{\text {recoil }} \\
(\mathrm{MeV})\end{array}$ & $\sigma_{\mathrm{ER}}(\mathrm{nb})$ & $\rho^{2}$ & $\begin{array}{c}\mathrm{ME}_{\mathrm{MRTQF}} \\
\left(\mathrm{keV} / c^{2}\right)\end{array}$ & $\begin{array}{c}\mathrm{ME}_{\mathrm{AME} 16} \\
\left(\mathrm{keV} / c^{2}\right)\end{array}$ & $\begin{array}{c}\Delta m \\
\left(\mathrm{keV} / c^{2}\right)\end{array}$ & $\begin{array}{c}N_{\text {ion }} \\
(\mathrm{counts})\end{array}$ \\
\hline${ }^{246} \mathrm{Es}$ & ${ }^{232} \mathrm{Th}\left({ }^{19} \mathrm{~F}, 5 n\right)$ & $99.6,103$ & $7.5,7.8$ & $(800)[29]$ & $0.92574351(44)$ & $67812(109)(32)$ & $67900^{\#}\left(224^{\#}\right)$ & $-88(109)(32)$ & 33 \\
${ }^{251} \mathrm{Fm}$ & ${ }^{238} \mathrm{U}\left({ }^{18} \mathrm{O}, 5 n\right)$ & 93.9 & 6.9 & $4000[30]$ & $0.94458700(14)$ & $75996(34)(25)$ & $75954(15)$ & $42(34)(25)$ & 397 \\
${ }^{249} \mathrm{Md}$ & ${ }^{203} \mathrm{Tl}\left({ }^{48} \mathrm{Ca}, 2 n\right)$ & 215 & 41.1 & $(40)[29]$ & $0.93706792(89)$ & $77259(221)(26)$ & $77232^{\#}\left(205^{\#}\right)$ & $27(221)(26)$ & 14 \\
${ }^{250} \mathrm{Md}$ & ${ }^{205} \mathrm{Tl}\left({ }^{48} \mathrm{Ca}, 3 n\right)$ & 223 & 42.3 & $(200)[29]$ & $0.94083491(56)$ & $78472(138)(25)$ & $78630^{\#}\left(298^{\#}\right)$ & $-158(138)(25)$ & 29 \\
${ }^{251} \mathrm{Md}$ & ${ }^{205} \mathrm{Tl}\left({ }^{48} \mathrm{Ca}, 2 n\right)$ & 215 & 40.8 & $760[31]$ & $0.94459923(24)$ & $79025(60)(23)$ & $78967(19)$ & $58(60)(23)$ & 173 \\
${ }^{252} \mathrm{Md}$ & ${ }^{238} \mathrm{U}\left({ }^{19} \mathrm{~F}, 5 n\right)$ & 98.6 & 7.3 & $(500)[29]$ & $0.94836715(36)$ & $80467(89)(22)$ & $80511^{\#}\left(130^{\#}\right)$ & $-44(89)(22)$ & 63 \\
${ }^{2549} \mathrm{No}$ & ${ }^{208} \mathrm{~Pb}\left({ }^{48} \mathrm{Ca}, 2 n\right)$ & 219 & 41.1 & $2000[32]$ & $0.95590832(17)$ & $84675(42)(19)$ & $84723.4(9.3)$ & $-48(42)(19)$ & 398 \\
\hline \hline
\end{tabular}

SHIPTRAP values for the masses of ${ }^{255} \mathrm{Lr}$ and ${ }^{255} \mathrm{No}$; this work provides the first direct mass measurements of ${ }^{251} \mathrm{Fm}$ and ${ }^{251} \mathrm{Md}$. In the cases of ${ }^{246} \mathrm{Es}$ and ${ }^{249,250,252} \mathrm{Md}$, no previous experimental mass data exist; however, our values are consistent with extrapolated mass values in AME16 with similar or higher mass precisions.

One important test applied to theoretical models is their ability to reproduce the shell gap parameter $\delta_{2 n}$ [35]. The shell gap parameter $\delta_{2 n}$ is calculated as

$$
\begin{aligned}
\delta_{2 n}(N, Z) & =S_{2 n}(N, Z)-S_{2 n}(N+2, Z) \\
& =2 B(N, Z)-B(N-2, Z)-B(N+2, Z),
\end{aligned}
$$

where $S_{2 n}(N, Z)$ and $B(N, Z)$ are the two-neutron separation energy and the total binding energy of nuclide ${ }^{N+Z} Z$. Newly determined $\delta_{2 n}(N, Z)$ values around $N=152$ for mendelevium and lawrencium are compared with the theoretical values in Fig. 7. As described in Eq. (1),

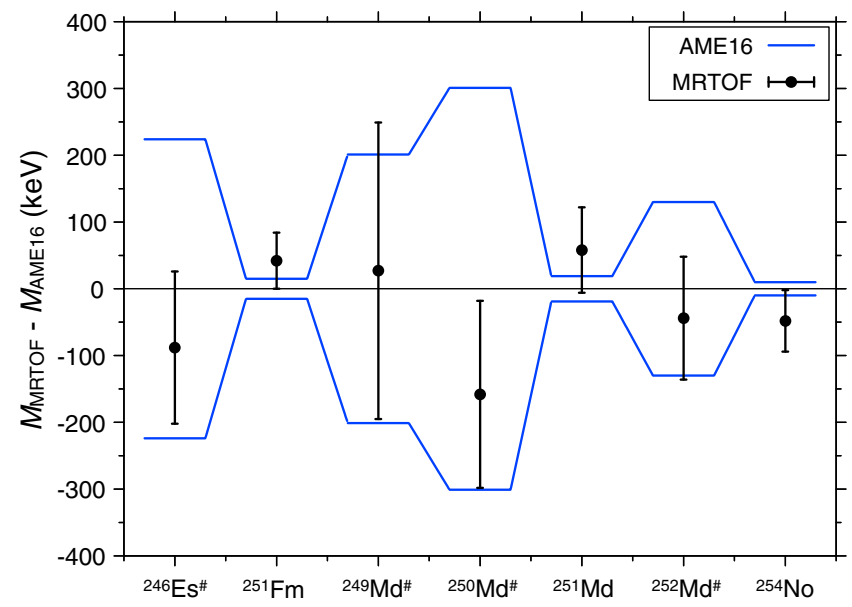

FIG. 6. Deviations between mass values determined in this work and AME16 [33] values. Error bars indicate $1 \sigma$ standard uncertainty of our data, while solid lines indicate the uncertainty of AME16. Isotopes designated with a superscript \# have extrapolated mass values in AME16. three isotopic masses- $M(N, Z), \quad M(N-2, Z)$, and $M(N+2, Z)$-are necessary to derive $\delta_{2 n}$. Using the new mass data in this work, we can make such a comparison between the experimental and theoretical values for $\mathrm{Md}$ and $\mathrm{Lr}$ in the vicinity of the $N=152$ subshell closure. For a comparison to the theory, we have selected global mass models representative of various common theoretical techniques: a shell model (DZ10 [36]), macroscopic-microscopic model (FRDM12 [37]
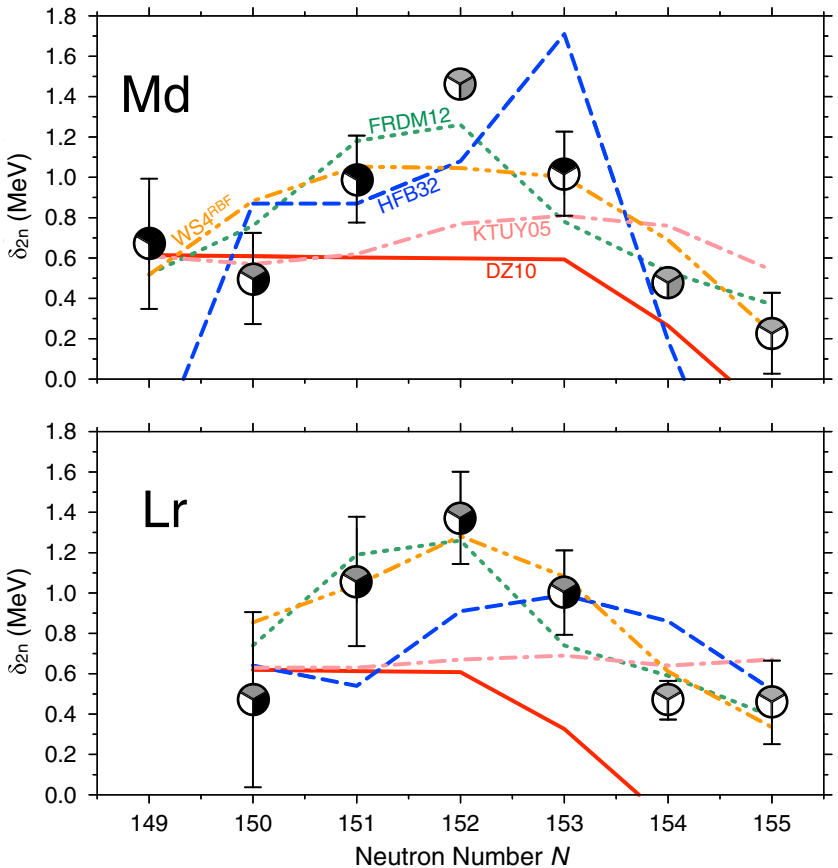

FIG. 7. Plots of empirical shell gap $\delta_{2 n}$ for $\mathrm{Md}$ and Lr isotopes. Data points are divided into three sections to indicate the contributions of the three mass values used in each. White indicates the extrapolated value from AME16, gray indicates the experimental value from AME16, and black indicates the experimental values from this work. Lines indicate results of theoretical models. 
TABLE II. Indirect determination of the mass excess (ME) using $\alpha$-decay $Q$ values $\left(Q_{\alpha}\right)$ taken from AME16 [28], along with the AME16 mass excesses $\left(\mathrm{ME}_{\mathrm{AME} 16}\right)$ and our deviation $(\Delta m)$ from them.

\begin{tabular}{lcccc}
\hline \hline & $\begin{array}{c}Q_{\alpha} \\
\text { Isotope } \\
\left(\mathrm{keV} / c^{2}\right)\end{array}$ & $\begin{array}{c}\mathrm{ME}_{\mathrm{MRTOF}}^{\text {Indirect }} \\
\left(\mathrm{keV} / c^{2}\right)\end{array}$ & $\begin{array}{c}\mathrm{ME}_{\mathrm{AME} 16} \\
\left(\mathrm{keV} / c^{2}\right)\end{array}$ & $\Delta m(\mathrm{keV})$ \\
\hline${ }^{253} \mathrm{Lr}$ & $8918(20)$ & $88602(222)$ & $88575^{\#}\left(202^{\#}\right)$ & $27(222)$ \\
${ }^{254} \mathrm{Lr}$ & $8816(12)$ & $89713(141)$ & $89871^{\#}\left(301^{\#}\right)$ & $-158(141)$ \\
${ }^{257} \mathrm{Db}$ & $9207(20)$ & $100234(224)$ & $100206^{\#}\left(203^{\#}\right)$ & $28(224)$ \\
${ }^{258} \mathrm{Db}$ & $9500(50)$ & $101638(149)$ & $101797^{\#}\left(306^{\#}\right)$ & $-159(149)$ \\
${ }^{261} \mathrm{Bh}$ & $10500(50)$ & $113158(229)$ & $113134^{\#}\left(209^{\#}\right)$ & $25(229)$ \\
${ }^{262} \mathrm{Bh}$ & $10319(15)$ & $114382(150)$ & $114541^{\#}\left(306^{\#}\right)$ & $-159(150)$ \\
${ }^{266} \mathrm{Mt}$ & $10996(25)$ & $127803(152)$ & $127962^{\#}\left(306^{\#}\right)$ & $-159(152)$ \\
\hline \hline
\end{tabular}

and $\mathrm{WS} 4^{\mathrm{RBF}}[38]$ ), a self-consistent mean-field model (HFB32 [39]), and a phenomenological mass model (KTUY05 [40]). Both macroscopic-microscopic mass models (FRDM12 and $\mathrm{WS} 4^{\mathrm{RBF}}$ ) reasonably predict the experimentally determined $\delta_{2 n}$ trends, although the peaking at $N=152$ is best reproduced by the FRDM12 model. HFB32 and KTUY05 peak beyond $N=152$, while DZ10 shows a flat trend with no peak. For lawrencium, WS4 ${ }^{\mathrm{RBF}}$ agrees well with both the general trend and the peak at $N=152$.

The location of the island of stability remains ambiguous. While experimental mass measurements of nuclei located within the hot-fusion superheavy island including the next deformed shell closure predicted at $N=162$ would be particularly valuable for this, in general, more experimentally determined masses in the transuranium region will allow for the improved extrapolation of mass values into the presumed region of the island of stability. By supplementing our precision, direct mass measurements with $\alpha$-decay $Q$ values, we can provide mass values for nuclei up to ${ }^{266} \mathrm{Mt}$, as listed in Table II. For future efforts to approach the island of stability, reliable theoretical predictions are crucial. Figure 8 shows the deviations between the various theoretical models and our experimental values. The best average agreement, with a mean deviation below

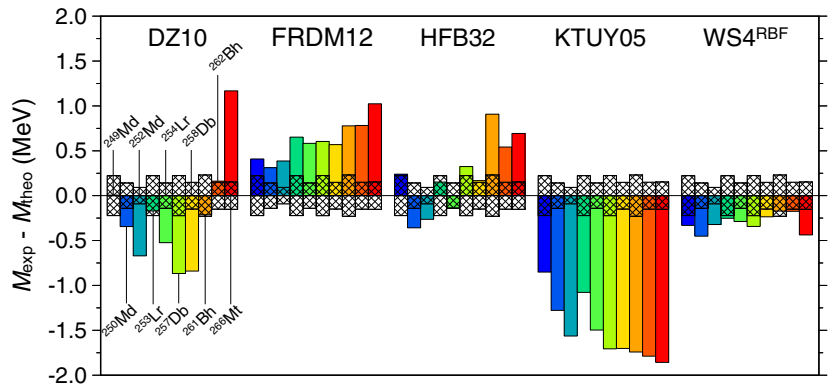

FIG. 8. Comparison of experimental masses with mass models. Each bar in a model corresponds to an isotope whose mass was determined for the first time in this work: ${ }^{249,250,252} \mathrm{Md},{ }^{253,254} \mathrm{Lr}$, ${ }^{257,258} \mathrm{Db},{ }^{261,262} \mathrm{Bh}$, and ${ }^{266} \mathrm{Mt}$. Meshed bars indicate experimentally determined mass uncertainties.
$500 \mathrm{keV} / c^{2}$, is obtained from the $\mathrm{WS} 4^{\mathrm{RBF}}$ mass model, which is based on the WS4 mass model [41] using a radial basis function approach for prediction. Except for the case of the KTUY05 model, a general agreement within about $1 \mathrm{MeV} / c^{2}$ is observed, although the deviations tend to increase with the mass.

In this study, we have directly measured the masses of ${ }^{246} \mathrm{Es},{ }^{251} \mathrm{Fm},{ }^{249-252} \mathrm{Md}$, and ${ }^{254 \mathrm{~g}, \mathrm{ml}} \mathrm{No}$. In the cases of ${ }^{254 \mathrm{~g} N o},{ }^{251} \mathrm{Fm}$, and ${ }^{251} \mathrm{Md}$, the AME16 mass values were derived from Penning trap data; the excellent agreement of our measurement with these Penning-trap-derived data provides a high degree of confidence in our experimental technique. Combining these results with $\alpha$-decay $Q$ values, the masses of ${ }^{253,254} \mathrm{Lr},{ }^{257,258} \mathrm{Db},{ }^{261,262} \mathrm{Bh}$, and ${ }^{266} \mathrm{Mt}$ could be indirectly determined. Particularly robust agreement is seen with the $\mathrm{WS} 4^{\mathrm{RBF}}$ mass model.

This work demonstrates the ability to perform direct mass measurements of both cold- and hot-fusion products, even with low recoil energy products $\left(E_{\text {recoil }} \approx 7 \mathrm{MeV}\right.$ ), by coupling a gas cell with GARIS-II. This technique could be applied to most nuclei produced with fusion-evaporation reactions in the SHN region. The overall system efficiency behind GARIS-II, excluding GARIS-II efficiency, from stopping in the gas cell to detection, was $\sim 2 \%$ limited by the double trap system. In the near future, modification to a single trap setup at a new experimental location should provide an improved system efficiency of more than $10 \%$ and a shorter measurement time. This will allow us to measure the masses of hot-fusion SHN having cross sections on the order of $10 \mathrm{pb}$.

We express gratitude to the Nishina Center for Accelerator-based Research at RIKEN and the Center for Nuclear Science at the University of Tokyo for their support during the online experiments. This work was financially supported by the Japan Society for the Promotion of Science KAKENHI (Grants No. 2200823, No. 24224008, No. 24740142, No. 15H02096, No. 15K05116, and No. 17H06090).

*Present address: Department of Physics, McGill University, Montreal, Quebec H3A 2T8, Canada. yito@physics.mcgill.ca

[1] D. Lunney, J. M. Pearson, and C. Thibault, Rev. Mod. Phys. 75, 1021 (2003).

[2] O. Sorlin and M.-G. Porquet, Prog. Part. Nucl. Phys. 61, 602 (2008).

[3] A. Sobiczewski, F. Gareev, and B. Kalinkin, Phys. Lett. 22, 500 (1966).

[4] Z. Patyk, A. Sobiczewski, P. Armbruster, and K.-H. Schmidt, Nucl. Phys. A491, 267 (1989).

[5] Z. Patyk and A. Sobiczewski, Nucl. Phys. A533, 132 (1991).

[6] G. I. Seaborg, Contemp. Phys. 28, 33 (1987).

[7] M. Bender, W. Nazarewicz, and P.-G. Reinhard, Phys. Lett. B 515, 42 (2001). 
[8] S. Cwiok, P.-H. Heenen, and W. Nazarewicz, Nature (London) 433, 705 (2005).

[9] Y. A. Lazarev, Y. V. Lobanov, Y. T. Oganessian, V. K. Utyonkov, F. S. Abdullin, A. N. Polyakov, J. Rigol, I. V. Shirokovsky, Y. S. Tsyganov, S. Iliev, V. G. Subbotin, A. M. Sukhov, G. V. Buklanov, B. N. Gikal, V. B. Kutner, A. N. Mezentsev, K. Subotic, J. F. Wild, R. W. Lougheed, and K. J. Moody, Phys. Rev. C 54, 620 (1996).

[10] Y. T. Oganessian, Radiochim. Acta 99, 429 (2011).

[11] D. Rudolph et al., Phys. Rev. Lett. 111, 112502 (2013).

[12] R. Julin, Nucl. Phys. A685, 221 (2001).

[13] D. Bucurescu and N. V. Zamfir, Phys. Rev. C 87, 054324 (2013).

[14] M. Block et al., Nature (London) 463, 785 (2010).

[15] E. M. Ramirez et al., Science 337, 1207 (2012).

[16] Y. Ito, P. Schury, M. Wada, S. Naimi, T. Sonoda, H. Mita, F. Arai, A. Takamine, K. Okada, A. Ozawa, and H. Wollnik, Phys. Rev. C 88, 011306 (2013).

[17] P. Schury, M. Wada, Y. Ito, F. Arai, S. Naimi, T. Sonoda, H. Wollnik, V. Shchepunov, C. Smorra, and C. Yuan, Nucl. Instrum. Methods Phys. Res., Sect. B 335, 39 (2014).

[18] D. Kaji, K. Morimoto, N. Sato, A. Yoneda, and K. Morita, Nucl. Instrum. Methods Phys. Res., Sect. B 317, 311 (2013).

[19] M. Wada, Y. Ishida, T. Nakamura, Y. Yamazaki, T. Kambara, H. Ohyama, Y. Kanai, T. M. Kojima, Y. Nakai, N. Ohshima, A. Yoshida, T. Kubo, Y. Matsuo, Y. Fukuyama, K. Okada, T. Sonoda, S. Ohtani, K. Noda, H. Kawakami, and I. Katayama, Nucl. Instrum. Methods Phys. Res., Sect. B 204, 570 (2003).

[20] G. Bollen, Int. J. Mass Spectrom. 299, 131 (2011).

[21] F. Arai, Y. Ito, M. Wada, P. Schury, T. Sonoda, and H. Mita, Int. J. Mass Spectrom. 362, 56 (2014).

[22] F. Arai, Y. Ito, I. Katayama, P. Schury, T. Sonoda, M. Wada, and H. Wollnik, J. Phys. Soc. Jpn. Conf. Proc. 6, 030110 (2015).

[23] N. E. Bradbury and R. A. Nielsen, Phys. Rev. 49, 388 (1936).

[24] Y. Ito, P. Schury, M. Wada, S. Naimi, C. Smorra, T. Sonoda, H. Mita, A. Takamine, K. Okada, A. Ozawa, and H. Wollnik, Nucl. Instrum. Methods Phys. Res., Sect. B 317, 544 (2013).

[25] A. L. Stancik and E. B. Brauns, Vib. Spectrosc. 47, 66 (2008).
[26] R Development Core Team, $R$ : A Language and Environment for Statistical Computing (R Foundation for Statistical Computing, Vienna, 2008), ISBN: 3-900051-07-0.

[27] D. Kaji and K. Morimoto, Nucl. Instrum. Methods Phys. Res., Sect. A 792, 11 (2015).

[28] W. Huang, G. Audi, M. Wang, F. Kondev, S. Naimi, and X. Xu, Chin. Phys. C 41, 030002 (2017).

[29] V. I. Zagrebaev, A. S. Denikin, A. V. Karpov, A. P. Alekseev, M. A. Naumenko, V. A. Rachkov, V. V. Samarin, and V. V. Saiko, Monte-Carlo code of NRV, http://nrv.jinr.ru/.

[30] E. D. Donets, V. A. Shchegolev, and V. A. Ermakov, Sov. J. Nucl. Phys. 2, 723 (1966).

[31] A. Chatillon et al., Phys. Rev. Lett. 98, 132503 (2007).

[32] Y. T. Oganessian, V. K. Utyonkov, Y. V. Lobanov, F. S. Abdullin, A. N. Polyakov, I. V. Shirokovsky, Y. S. Tsyganov, A. N. Mezentsev, S. Iliev, V. G. Subbotin, A. M. Sukhov, K. Subotic, O. V. Ivanov, A. N. Voinov, V. I. Zagrebaev, K. J. Moody, J. F. Wild, N. J. Stoyer, M. A. Stoyer, and R. W. Lougheed, Phys. Rev. C 64, 054606 (2001).

[33] M. Wang, G. Audi, F. G. Kondev, W. J. Huang, S. Naimi, and X. Xu, Chin. Phys. C 41, 030003 (2017).

[34] F. P. Heßberger, S. Antalic, B. Sulignano, D. Ackermann, S. Heinz, S. Hofmann, B. Kindler, J. Khuyagbaatar, I. Kojouharov, P. Kuusiniemi, M. Leino, B. Lommel, R. Mann, K. Nishio, A. G. Popeko, S. Saro, B. Streicher, J. Uusitalo, M. Venhart, and A. V. Yeremin, Eur. Phys. J. A 43, 55 (2010).

[35] K. Rutz, M. Bender, T. Bürvenich, T. Schilling, P.-G. Reinhard, J. A. Maruhn, and W. Greiner, Phys. Rev. C 56, 238 (1997).

[36] J. Duflo and A. P. Zuker, Phys. Rev. C 52, R23 (1995).

[37] P. Möller, A. Sierk, T. Ichikawa, and H. Sagawa, At. Data Nucl. Data Tables 109, 1 (2016).

[38] N. Wang and M. Liu, Phys. Rev. C 84, 051303 (2011).

[39] S. Goriely, N. Chamel, and J. M. Pearson, Phys. Rev. C 93, 034337 (2016).

[40] H. Koura, T. Tachibana, M. Uno, and M. Yamada, Prog. Theor. Phys. 113, 305 (2005).

[41] N. Wang, M. Liu, X. Wu, and J. Meng, Phys. Lett. B 734, 215 (2014). 